\title{
Fecal microbiota profiling in irritable bowel syndrome and inflammatory bowel disease patients with irritable bowel syndrome-type symptoms
}

\author{
Xiufang Cui ${ }^{1,2+}$, Haiyang Wang ${ }^{3 \dagger}$, Ziping Ye ${ }^{1}$, Yi Li $^{3}$, Xinyun Qiu ${ }^{1}$ and Hongjie Zhang ${ }^{1 *}$
}

\begin{abstract}
Background: The intestinal microbiota is thought to be involved in the occurrence of inflammatory bowel disease in remission with irritable bowel syndrome (IBS)-type symptoms, but the specific distinct profile of these bacteria remains unclear. This cross-sectional study aims to investigate the fecal microbiota profiling in patients with these diseases.

Methods: Fecal samples from 97 subjects, including Crohn's disease patients in remission with IBS-type symptoms $\left(\mathrm{CDR}^{\left.-\mathrm{BSS}^{+}\right)}\right.$or without IBS-type symptoms $\left(\mathrm{CDR}-\mathrm{IBS}^{-}\right)$, ulcerative colitis patients in remission with IBS-type symptoms $\left(\mathrm{UCR}^{-I B S}{ }^{+}\right)$or without IBS-type symptoms (UCR-IBS $\left.{ }^{-}\right)$, IBS patients and healthy controls, were collected and applied $16 \mathrm{~S}$ ribosomal DNA (rDNA) gene sequencing. The $\mathrm{V} 4$ hypervariable regions of $16 \mathrm{~S}$ rDNA gene were amplified and sequenced by the lllumina MiSeq platform. The differences in the sample diversity index in groups were analyzed with R software.
\end{abstract}

Results: The richness of the intestinal microbiota in the CDR-IBS group was markedly lower than those in the control and IBS groups based on the analysis of observed species and the Chao index $(P<0.05)$. The observed species index in the CDR-IBS ${ }^{+}$group was higher than that in the CDR-IBS ${ }^{-}$group (median index: 254.8 vs 203, $P=0.036$ ). No difference was found in alpha diversity between UCR patients with IBS-type symptoms and those without related symptoms. At the genus level, the number of Faecalibacterium in CDR patients with IBS-type symptoms increased significantly, while Fusobacterium decreased versus those without such symptoms (mean relative abundance of Faecalibacterium: $20.35 \%$ vs $5.18 \%, P<0.05$; Fusobacterium: $1.51 \%$ vs $5.2 \%, P<0.05$ ). However, compared with the UCR-IBS ${ }^{-}$group, the number of Faecalibacterium in the UCR-IBS ${ }^{+}$group decreased, while the number of Streptococcus increased, but there was no significant difference in the genus structure. The abundance and composition of the microbiota of IBS patients were not distinct from those of healthy controls.

Conclusions: The IBS-type symptoms in CD patients in remission may be related to an increase in Faecalibacterium and a decrease in Fusobacterium. The IBS-type symptoms in UC patients in remission cannot be explained by changes in the abundance and structure of the intestinal microbiota.

\footnotetext{
*Correspondence: hjzhang06@163.com

${ }^{+}$Xiufang Cui and Haiyang Wang contributed equally to this article

1 Department of Gastroenterology, First Affiliated Hospital of Nanjing

Medical University, 300\# Guangzhou Road, Nanjing 210029, Jiangsu

Province, People's Republic of China

Full list of author information is available at the end of the article
}

(C) The Author(s) 2021. Open Access This article is licensed under a Creative Commons Attribution 4.0 International License, which permits use, sharing, adaptation, distribution and reproduction in any medium or format, as long as you give appropriate credit to the original author(s) and the source, provide a link to the Creative Commons licence, and indicate if changes were made. The images or other third party material in this article are included in the article's Creative Commons licence, unless indicated otherwise in a credit line to the material. If material is not included in the article's Creative Commons licence and your intended use is not permitted by statutory regulation or exceeds the permitted use, you will need to obtain permission directly from the copyright holder. To view a copy of this licence, visit http://creativecommons.org/licenses/by/4.0/. The Creative Commons Public Domain Dedication waiver (http://creativeco mmons.org/publicdomain/zero/1.0/) applies to the data made available in this article, unless otherwise stated in a credit line to the data. 
Keywords: Inflammatory bowel disease, Intestinal microbiota, Irritable bowel syndrome (IBS)-type symptoms

\section{Background}

Changes in the intestinal microbiota can result in the loss of intestinal homeostasis, which has been found in a variety of intestinal disorders, including inflammatory bowel disease (IBD) and irritable bowel syndrome (IBS) [1]. IBD is a chronic relapsing inflammatory disease of the gastrointestinal tract with unknown etiology and includes Crohn's disease (CD) and ulcerative colitis (UC). The pathogenesis of IBD remains incompletely understood, though it is currently recognized that it is closely related to genetic susceptibility, environment, a disruption of the intestinal microbiota, and immune disorders, especially of the intestinal microbiota. A large number of studies have confirmed that the interaction between intestinal flora and genetic susceptibility can be considered a contributor to the pathogenesis of IBD by triggering an exacerbated immune response [2]. Previous studies indicate that altered gut microbiota composition was found in IBD patients, including reductions in microbial diversity and richness [3]. Specifically, beneficial bacteria decrease, while harmful bacteria increase $[4,5]$.

Irritable bowel syndrome (IBS) is a functional bowel disease characterized by recurrent abdominal pain, bloating, and altered bowel habits. IBD patients in the active stage often have abdominal pain, diarrhea, bloody stools and other uncomfortable symptoms. Some IBD patients in remission (IBDR) have persistent gastrointestinal symptoms, including abdominal pain, diarrhea and abdominal discomfort. For IBDR, these symptoms meet the criteria for IBS and can be defined as IBS-type symptoms [6-8]. According to previous studies, the prevalence of IBS-type symptoms in patients with IBD with clinically quiescent disease ranges from 25 to $60 \%$ due to the different definitions of remission and population sizes $[9,10]$. There is a lack of evidence-based therapeutic options available for the management of such patients, who experience a reduced quality of life equivalent to that of patients with overt inflammatory disease activity [10].

Although IBS and IBD are functional and organic diseases, respectively, they have some common etiologies, especially the intestinal microbiome [11, 12]. Emerging evidence suggests an important role of the intestinal microbiota in the pathophysiology of IBS $[13,14]$. Previous observational studies have shown that intestinal infections may cause IBS, and probiotics can be used to treat IBS $[15,16]$. The etiology of IBS-type symptoms in IBDR patients is still unclear and remains controversial. In addition, at present, there is still a lack of knowledge and effective treatment of the disease in patients with IBDR accompanied by IBS symptoms.

Therefore, we hypothesized that the IBS-type symptoms of IBD patients in remission would be closely related to alterations in the intestinal microbiota. To clarify this hypothesis, we performed a cross-sectional study to initially explore the alterations in the intestinal microbiota of IBD patients in remission with IBS-type symptoms.

\section{Methods \\ Participants and setting IBD patients}

All participants with IBD had an established radiological, histological, or endoscopic diagnosis of $\mathrm{CD}$ or UC according to the criteria of the European Crohn \& Colitis Organization (ECCO) [17]. In this study, IBD patients in clinical remission were included, and the inclusion criteria were as follows [18]: (1) Mayo score $\leqq 2$ and singleitem score $<1$; (2) Crohn's disease activity index (CDAI) $\leqq 150$; and (3) participants who did not take antibiotics, probiotics, colon cleansing liquids, and similar remedies at least 2 months prior to the study. Exclusion criteria included an inability to understand the Chinese version of the informed consent form, uncategorized IBD, a history of gastroenterology surgery, and female subjects who were lactating or pregnant.

\section{IBS and IBD-IBS patients}

IBS was evaluated by excluding organic diseases on the basis of Rome IV diagnostic items as follows [19]. Patients with confirmed IBD had symptoms of abdominal pain and changes in bowel habits, and these symptoms met the Rome IV criteria [19] and were defined as IBD with IBS-type symptoms (IBD-IBS) according to previous studies $[20,21]$.

\section{Healthy controls}

Normal healthy controls were selected from healthy people in the physical examination center. The data were collected from the First Affiliated Hospital of Nanjing Medical University from August 2018 to September 2019.

\section{Sample collection and genomic DNA extraction}

We collected fresh fecal samples from all enrolled subjects and immediately stored them in a $-80{ }^{\circ} \mathrm{C}$ refrigerator within $2 \mathrm{~h}$ to avoid bacterial overgrowth in an oxygen environment. According to the manufacturer's instructions, genomic DNA for microbiome analysis 
was extracted using a special QIAamp DNA Stool Mini Kit (Qiagen, Hilden, Germany). Briefly, fecal samples (200 $\mathrm{mg}$ ) were added to a $2 \mathrm{ml}$ microcentrifuge tube, and tube wea was placed on ice. One milliliter of InhibitEX buffer was added to each fecal sample, and the tube was vortexed continuously for $1 \mathrm{~min}$ until the sample was thoroughly homogenized. The subsequent extraction protocol was carried out in strict compliance with recommendations from the QIAamp DNA Stool Mini Kit instructions.

\section{Sequencing}

All DNA samples were quality controlled before being amplified by polymerase chain reaction (PCR) of the V4 hypervariable region of the $16 \mathrm{~S}$ rRNA gene, a reliable indicator of bacterial taxonomy [22]. Diluted genomic DNA was used as a template, and amplification PCR was performed using specific primers with barcodes, Phusion ${ }^{\circledR}$ High-Fidelity PCR Master Mix with GC Buffer from New England Biolabs, and high-efficiency and high-fidelity enzymes according to the selection of the sequencing region. PCR products were mixed and purified using a Qiagen Gel Extraction Kit. Magnetic beads were used to screen the target amplicon fragments, and finally, the qualified library was used for cluster preparation and paired-end sequencing through the Illumina platform (HiSeq or MiSeq) following the manufacturer's instructions.

\section{Data collection and analysis}

Samples were merged to build a library using barcodes, and after obtaining clean data, the barcode sequences were used to split the samples through an internally written program. The allowed number of mismatches between barcode sequences and sequencing reads was 0 bp. Using this method, paired-end sequencing was performed on an Illumina platform (HiSeq or MiSeq), and the low-quality reads were removed from the offmachine data. Paired end reads were spliced into tags through the overlap relationship between reads using FLASH (V1.2.11, http://ccb.jhu.edu/software/FLASH/) [23]. The low-quality raw tags were removed, and the high-quality tags remained for subsequent analysis according to QIIME (V1.8.0, http://qiime.org/index. html) [24]. Finally, the obtained effective clean tags were analyzed using the UCHIME algorithm (UCHIME Algorithm, v7.0.1090, http://www.drive5). The software USEARCH (v7.0.1090) was used to cluster the spliced tags into operational taxonomic units (OTUs), with the following procedure: 1) UPARSE was used to perform clustering at $97 \%$ similarity to obtain the representative sequences of OTUs; 2) UCHIME (v4.2.40) [4] was used to remove the chimeras generated by PCR amplification from the OTU representative sequence by comparing the sequences with the reference database (Gold database, v20110519, http://drive5.Com/uchime/uchime_downl oad.html; UNITE, v20140703); 3) the Usearch_global method was used to align all tags back to the OTU representative sequences and obtain the abundance statistics table of each sample in each OTU. After obtaining the representative sequences of OTUs, they were compared with the database Greengene_2013_5_99 through RDP Classifier (v2.2) software, the species were annotated, and the confidence threshold was set to 0.6. After the spliced tags were optimized, all samples were selected with the smallest number of tags and clustered into operational taxonomic units (OTUs) for species classification at $97 \%$ similarity. In the abundance information, the abundance of OTUs preliminarily indicates the species richness of the sample. In this study, the alpha diversity value of the sample was calculated using mothur (v1.31.2) software. The differences in the sample diversity index in groups were analyzed and displayed with R (v3.1.1) software based on the standardized output data.

\section{Statistical analysis}

All the data were analyzed using Statistical Package for Social Sciences version 25.0 (IBM Company, Armonk, NY). Age parameter data are expressed as the mean \pm standard deviation. Unless specifically explained, the majority of microbiota data were nonnormally distributed, and the data are expressed as the median (maximum, minimum). Kruskal-Wallis one-way analysis of variance was used to compare the microbiota data. Partial graphs were drawn using GraphPad software 8.0 (GraphPad Inc., San Diego, CA). A $P$ value lower than 0.05 was considered statistically significant.

\section{Results}

\section{Demographics and clinical characteristics of the study subjects}

A total of 97 subjects were enrolled in the study, including 34 IBS patients, 45 IBD patients in remission, and 18 healthy controls. All subjects who met the enrollment criteria from the First Affiliated Hospital of Nanjing Medical University were recruited from August 2018 to September 2019. The mean ages were 42.9 years in the IBS group, 30.9 years in the CDR-IBS + group, 29.8 years in the CDR-IBS- group, 37.1 years in the UCR-IBS + group, 42.4 years in the UCR-IBS- group and 37.9 years in the control group. The proportion of male subjects was $54.6 \%$ (53/97). However, there were more female subjects in the IBS group $(61.7 \%, 21 / 34)$, which might be closely related to the obvious sex difference in the incidence of this kind of disease. Detailed demographic data and clinical characteristics of all included subjects are listed in Table 1. 
Table 1 Clinical characteristics of all included patients

\begin{tabular}{|c|c|c|c|c|c|c|}
\hline & Control & IBS & CDR-IBS + & CDR-IBS- & UCR-IBS+ & UCR-IBS- \\
\hline$n$ & 18 & 34 & 10 & 15 & 10 & 10 \\
\hline Age, mean $\pm S D$, yr & $37.9 \pm 8.3$ & $42.9 \pm 14.0$ & $30.9 \pm 13.9$ & $29.8 \pm 10.5$ & $37.1 \pm 13.1$ & $42.4 \pm 12.7$ \\
\hline Sex, male/female & $10 / 5$ & $13 / 21$ & $5 / 5$ & $11 / 4$ & $8 / 2$ & $8 / 2$ \\
\hline Disease duration, median (range) & & $12(6-240)$ & $24(12-60)$ & $24(10-120)$ & $27(12-120)$ & $27(9-120)$ \\
\hline \multicolumn{7}{|l|}{ Montreal A (Age of onset, yr), $n(\%)$} \\
\hline $\mathrm{A} 1(<16)$ & & & 0 & $1(6.7)$ & & \\
\hline $\mathrm{A} 2(17 \sim 40)$ & & & $8(80)$ & $12(80)$ & & \\
\hline $\mathrm{A} 3(>40)$ & & & $2(20)$ & $2(13.3)$ & & \\
\hline \multicolumn{7}{|l|}{ Montreal A (Location), n (\%) } \\
\hline L1 (ileal) & & & $3(30)$ & $5(33.3)$ & & \\
\hline L2 (colonic) & & & $4(40)$ & $3(20)$ & & \\
\hline L3 (ileocolonic) & & & $3(30)$ & $7(46.7)$ & & \\
\hline L4 (upper gastrointestinal tract) & & & 0 & 0 & & \\
\hline \multicolumn{7}{|l|}{ Montreal B (Behavior), n (\%) } \\
\hline B1 (nonstricturing, nonpenetrating) & & & $9(90)$ & 13(86.7) & & \\
\hline B2 (stricturing) & & & $1(10)$ & $1(6.7)$ & & \\
\hline B3 (penetrating) & & & 0 & $1(6.7)$ & & \\
\hline \multicolumn{7}{|l|}{ Montreal $E, n(\%)$} \\
\hline E1 (ulceration proctitis) & & & & & $3(30)$ & $3(30)$ \\
\hline E2 (left-sided ulceration colitis) & & & & & $3(30)$ & $4(40)$ \\
\hline E2 (extensive ulceration colitis) & & & & & $4(40)$ & $3(30)$ \\
\hline \multicolumn{7}{|l|}{ Therapy, $n(\%)$} \\
\hline $5-A S A$ & & & $2(20)$ & $1(6.7)$ & $8(80)$ & $7(70)$ \\
\hline Azathioprine & & & $3(30)$ & $2(13.3)$ & $0(0)$ & $0(0)$ \\
\hline Steroids & & & $0(0)$ & $0(0)$ & $2(20)$ & $3(30)$ \\
\hline Infliximab & & & $5(50)$ & $11(73.3)$ & $0(0)$ & $0(0)$ \\
\hline Nutritional treatment & & & $1(10)$ & $2(13.3)$ & $0(0)$ & $0(0)$ \\
\hline
\end{tabular}

CD: Crohn's disease, CDR: Crohn's disease in remission, CDR-IBS +: CDR with IBS-type symptoms, CDR-IBS-: CDR without IBS-type symptoms. UC: ulcerative colitis, UCR: ulcerative colitis in remission, UCR-IBS + : UCR with IBS-type symptoms, UCR-IBS-: UCR without IBS-type symptoms; SD: standard deviation; 5-ASA: 5-aminosalicylic acid

\section{Overall sequencing results}

The paired-end reads were optimized to remove lowquality reads and clustered into operational taxonomic units (OTUs) for species classification at 97\% similarity, and the abundance information of each sample in each OTU was counted. The abundance preliminarily explains the species richness of the sample. A total of 4,869,075 high-quality tags were obtained, and the average number of tags for each sample was 50,197. According to the 97\% similar clustering principle, a total of 1118 OTUs were generated from 97 samples. Compared with the control group, the numbers of OTUs in IBS, CD patients in remission with IBS-type symptoms $\left(\mathrm{CDR}-\mathrm{IBS}^{+}\right), \mathrm{CD}$ patients in remission without IBS-type symptoms (CDR$\mathrm{IBS}^{-}$), UC patients in remission with IBS-type symptoms $\left(\mathrm{UCR}^{-} \mathrm{IBS}^{+}\right)$and UC patients in remission without IBStype symptoms (UCR-IBS ${ }^{-}$) were reduced, but only the OTUs of patients in CDR-IBS ${ }^{-}$had a significant difference $(163.7 \pm 65.98$ vs $240.8 \pm 66.75, P<0.05)$, suggesting that this group of patients may have the lowest species abundance. The detailed results are shown in Table 2.

Table 2 The differences of the number of Tags and OUTS

\begin{tabular}{|c|c|c|c|c|c|c|}
\hline & Control & IBS & CDR-IBS $^{+}$ & $\mathrm{CDR}^{-\mathrm{IBS}^{-}}$ & UCR-IBS ${ }^{+}$ & UCR-IBS ${ }^{-}$ \\
\hline Tag number & $48,231 \pm 3293$ & $50,825 \pm 2820$ & $48,491 \pm 3337$ & $51,135 \pm 2373$ & $51,259 \pm 4455$ & $50,836 \pm 1891$ \\
\hline OUT numbers & $240.8 \pm 66.75$ & $221.4 \pm 70.84$ & $225.5 \pm 70.48$ & $163.7 \pm 65.98^{*}$ & $204.3 \pm 79.91$ & $213.2 \pm 78.62$ \\
\hline
\end{tabular}

CD: Crohn's disease, CDR: Crohn's disease in remission, CDR-IBS +: CDR with IBS-type symptoms, CDR-IBS-: CDR without IBS-type symptoms. UC: ulcerative colitis, UCR: ulcerative colitis in remission, UCR-IBS +: UCR with IBS-type symptoms, UCR-IBS-: UCR without IBS-type symptoms. Compared with HC, IBS, CDR-IBS+, ${ }^{*} P<0.05$ 


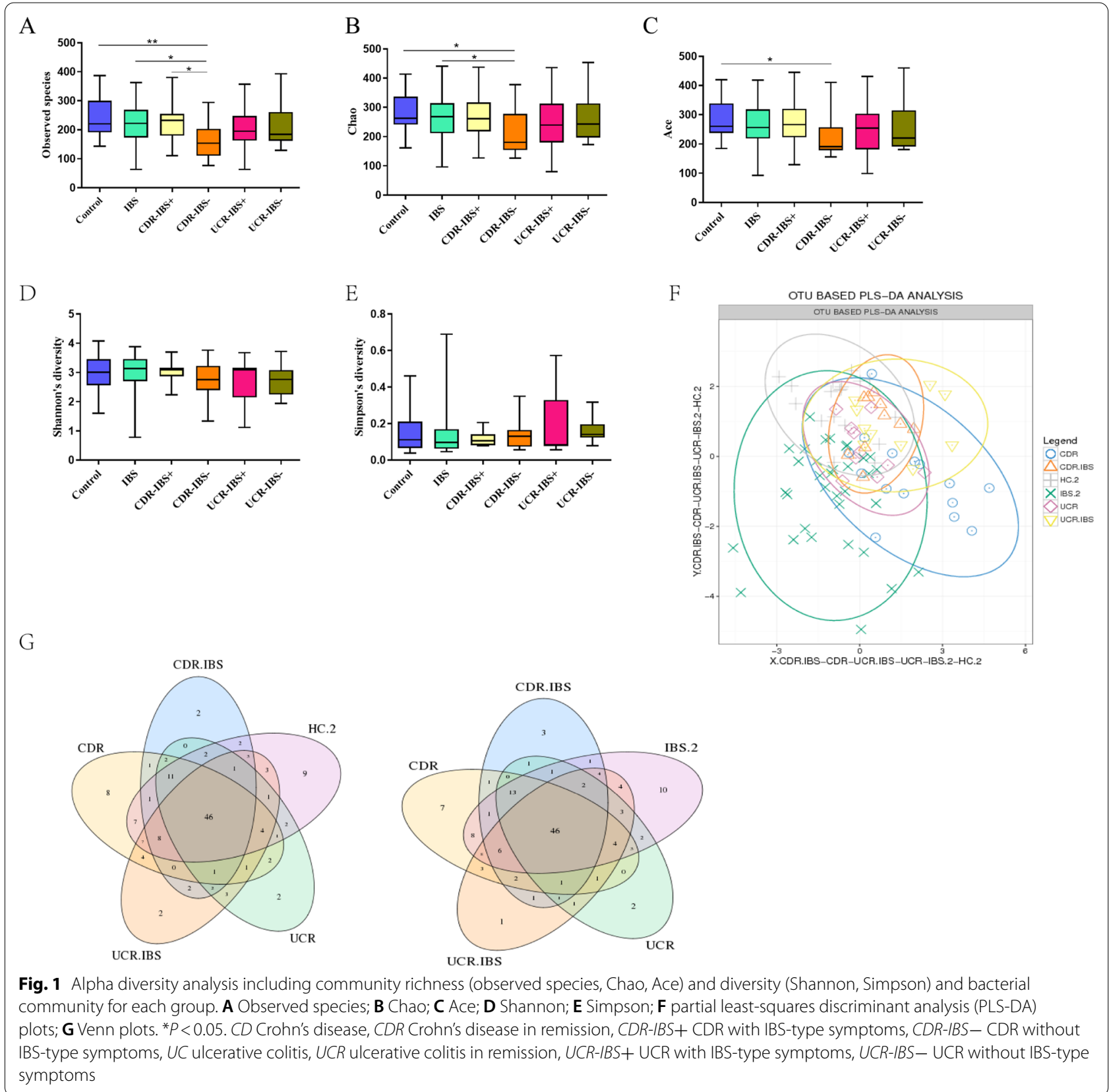

\section{Characteristics of the microbial diversity in different groups}

A rarefaction curve was used to reflect the rationality of the amount of sequencing data. A rarefaction curve was obtained for each sample according to the number of bacterial OTUs on sequence counts at different sequencing depths. As shown in Additional file 1: Fig. S1, the number of sequences continued to increase, and the rarefaction curve of each sample tended to be saturated, which indicated that the final sequencing data in the study were reliable. Alpha diversity was used to evaluate the differences in the microbiota of samples in the control, IBS, CDR-IBS ${ }^{+}, \mathrm{CDR}^{-\mathrm{IBS}^{-}}$, UCR-IBS ${ }^{+}$, and $\mathrm{UCR}-\mathrm{IBS}^{-}$ groups. The observed species index, Chao index and Ace index were calculated to reflect the species richness of the microbial community in the sample, while the Shannon index and Simpson index reflected species diversity, which was affected by the species richness and species evenness of the sample community. The results of the comparison among the alpha diversity index groups indicated that the observed species index and the Chao and Ace of CDR-IBS ${ }^{-}$indexes were significantly decreased 
compared with those of the control groups, while no difference was found among the other groups (Additional file 1: Fig. S1A-C). Meanwhile, the observed species of microbiota in the $\mathrm{CDR}^{-\mathrm{IBS}^{-}}$group was lower than that in the CDR-IBS ${ }^{+}$group. The Chao index in the CDRIBS $^{-}$group was lower than that in the IBS group. Therefore, the richness of microbiota in fecal samples from CDR-IBS $^{-}$groups was significantly decreased based on the analysis of alpha diversity. There were no differences in the Shannon index and the Simpson index among the six groups (Fig. 1D, E). However, there was no significant difference in the diversity analysis of patients among the IBS, CDR-IBS ${ }^{+}$and $\mathrm{UCR}^{-\mathrm{IBS}^{+}}$groups, including the observed species, Chaol index and ACE index. Furthermore, the overall microbiota structures were analyzed according to the number of shared or unique OTUs. The results are displayed in partial least-squares discriminant analysis (PLS-DA) plots and Venn plots (Fig. 1F, G) and heat maps at the phylum level. PLS-DA and heat maps indicated that the microbiota structure had slight differences among the groups. However, the majority of the bacterial communities among the six groups overlapped.

\section{Overall taxonomic compositions of the IBDR, IBS and control groups at the phylum}

As shown in Fig. 2, the proportions of different species at the phylum level are summarized in detail for each sample. The phylum levels of taxonomic composition in fecal samples of all groups with major microbiota were as follows: Firmicutes, Bacteroidetes, Proteobacteria, Actinobacteria, Fusobacteria, and Verrucomicrobia. (Fig. 2A, B). There was no significant difference in microbiota composition at the phylum level among the six groups. Compared with the control group, CDR patients with or without IBS-type symptoms had a decreasing trend in the abundance of Bacteroidetes, but there were no statistically significant differences. A relatively decreasing trend in the abundance of Bacteroidetes was found in the CDR groups compared with the IBS group (median proportional abundance CDR-IBS ${ }^{+}$vs IBS: $30.0 \%$ vs $47.6 \%$, CDR-IBS $^{-}$vs IBS: $34.7 \%$ vs $47.6 \%$, Fig. $2 C$ ), but no significant difference was obtained $(P>0.05)$. The proportion of Firmicutes displayed relative increasing trends in the $\mathrm{CDR}^{-\mathrm{IBS}^{+}}$and UCR-IBS ${ }^{+}$groups. No difference in Fusobacteria phylum abundance was determined in the current populations, although there was an increasing trend for IBDR in different types regardless of the presence or absence of IBS-type symptoms. Overall, there was no significant difference in microbiota among the IBS, CDR$\mathrm{IBS}^{+}$and UCR-IBS ${ }^{+}$groups. Further analysis showed that there was no significant difference in the alteration of the microbiota community at the phylum level between the
UCR with or without IBS-type symptoms and the CDR groups.

\section{Comparison of microbiota composition among the different groups at the genus level}

The overall genera from each sample are displayed in a bar plot of taxonomic analysis. The main microbiota compositions at the genus level were as follows: Bacteroides, Faecalibacterium, Prevotella, Escherichia, Roseburia, Blautia, Streptococcus, Fusobacterium, Hemophilus, and Lachnospira (Fig. 3A). There were no significant differences in the changes at the genus level between control and IBS subjects (Fig. 3B). The relative abundances of Bacteroides were slightly increased in IBS, UCR-IBS ${ }^{+}$and UCR-IBS ${ }^{-}$patients compared to that in controls, but the differences were not statistically significant. The mean abundance of Bacteroides tended to decrease in the $\mathrm{CDR}-\mathrm{IBS}^{+}$group and increase in the UCR-IBS ${ }^{+}$group compared with the IBS group, although the difference was not statistically significant. Compared with CDR-IBS ${ }^{-}$, the abundances of Faecalibacterium, Roseburia and Streptococcus tended to increase, while that of Prevotella, Escherichia, and Fusobacterium decreased in the CDR$\mathrm{IBS}^{+}$group, of which the number of Faecalibacterium was significantly higher. While that of Fusobacterium was lower in CDR-IBS ${ }^{+}$than in CDR-IBS ${ }^{-}$(mean relative abundance of Faecalibacterium: $20.35 \%$ vs $5.18 \%$, $P<0.05$; Fusobacterium: $1.51 \%$ vs $5.2 \%, P<0.05)$. In addition, the changes in the microbiota community in UCR subjects were not the same as those in CDR subjects. The results indicated that the abundances of Fusobacterium and Streptococcus were increased, but the abundances of Faecalibacterium, Escherichia, and Lachnospira were slightly decreased in the UCR-IBS ${ }^{+}$ group compared to the UCR-IBS ${ }^{-}$group, although none of the differences were statistically significant. Differences were also found between CDR and UCR at the genus level. There was a significantly greater abundance of Faecalibacterium in UCR-IBS ${ }^{-}$rela-

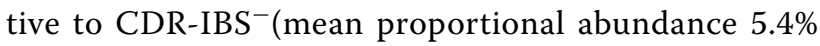
vs $16.6 \%, P=0.012$ ) and a greater abundance of Fusobacterium in $\mathrm{CDR}^{-\mathrm{IBS}^{-}}$relative to $\mathrm{UCR}^{-} \mathrm{IBS}^{-}$(mean proportional abundance $5.6 \%$ vs $0.04 \%, P=0.001$ ). The genus differences between CDR-IBS ${ }^{+}$and UCR-IBS ${ }^{+}$ patients were not statistically significant. The differences in the microbiota communities among the IBS, $\mathrm{CDR} \mathrm{IBS}^{+}$and $\mathrm{UCR}^{-} \mathrm{IBS}^{+}$groups were also analyzed. The results showed that the mean abundances of Faecalibacterium and Streptococcus tended to increase, while the levels of Prevotella and Lachnospira tended to decrease in the CDR-IBS ${ }^{+}$and UCR-IBS ${ }^{+}$groups 


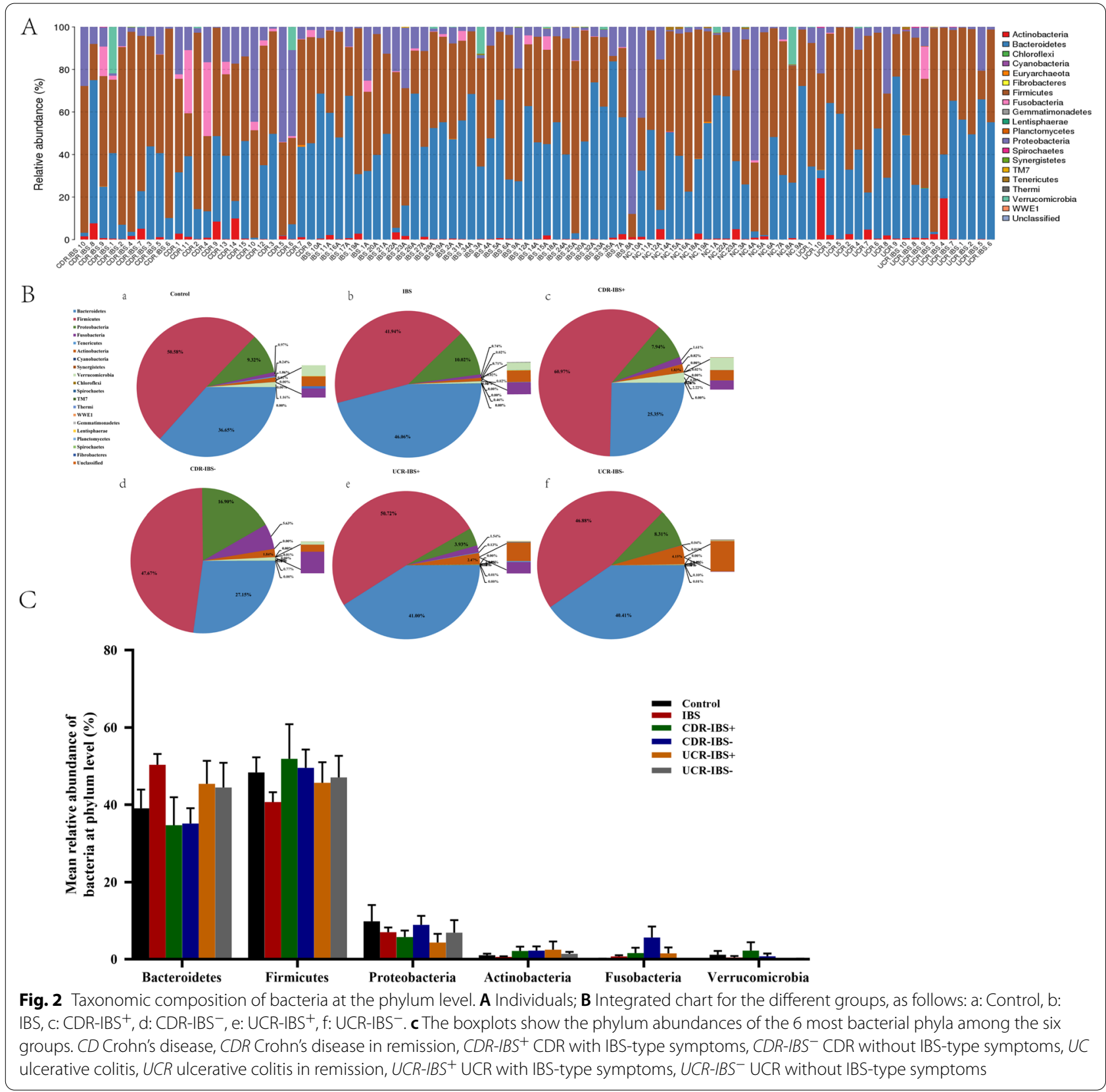

compared with the IBS groups. However, further analysis of the genera among IBS, CDR-IBS ${ }^{+}, \mathrm{UCR}^{-\mathrm{IBS}^{+}}$ patients did not reveal a statistically significant difference. (Fig. 3B). The differences in genera are displayed in detail in Table 3. As shown, other genera included Butyricimonas, Odoribacter, Enterococcus, Clostridium, Megasphaera, Ruminofilibacter, Gemmiger, Desulfovibrio, Actinomyces, and Akkermansia. The increasing and decreasing trends of relative abundance in genera are listed in Table 3.

\section{Discussion}

The gut microbiome contains more than 100 trillion different microorganisms, including bacteria, fungi, viruses and protozoa [25]. The majority of the intestinal bacteria belong to four phyla, including Firmicutes, Bacteroidetes, Proteobacteria and Actinobacteria, and in healthy adults, Firmicutes and Bacteroidetes are the main phyla [26]. A number of studies have confirmed that changes in the structure and abundance of the intestinal microbiota play important biological roles, including immune regulation, nutrition, metabolism and defense against pathogens 


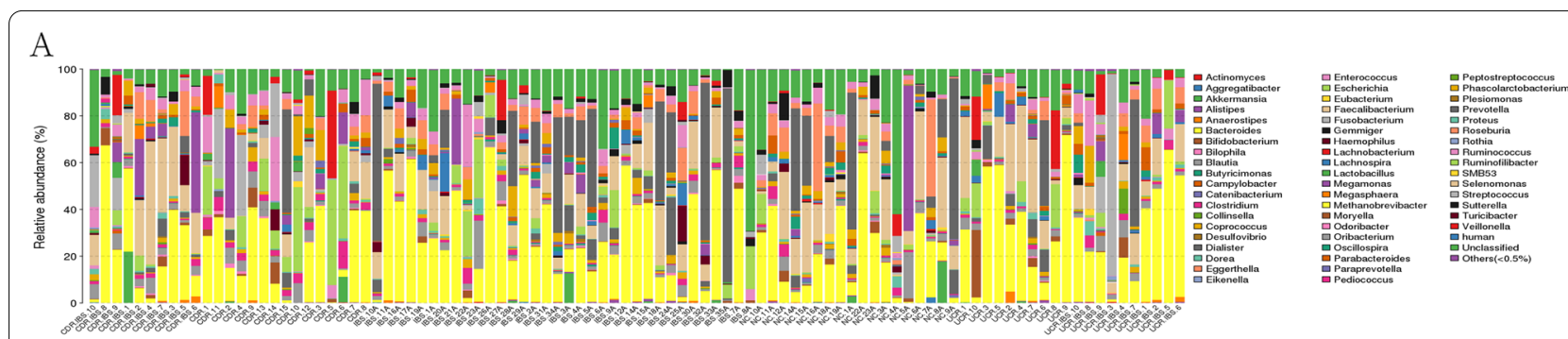

$\mathrm{B}$

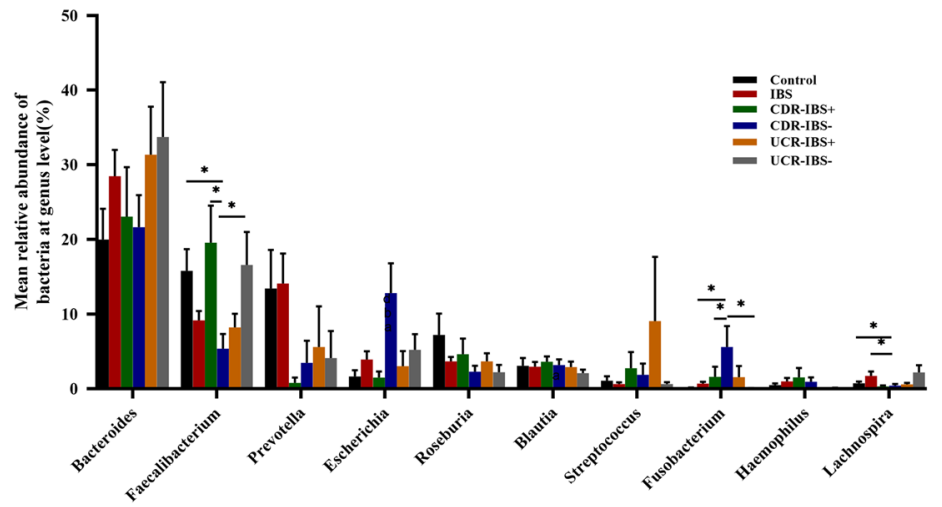

Fig. 3 Analysis of taxonomic composition at the genus level. A Individually; $\mathbf{B}$ the boxplot indicates the most abundant bacterial genera in different groups, including the Control, IBS, CDR-IBS ${ }^{+}, C^{2}$ CDR-IBS ${ }^{-}$, UCR-IBS ${ }^{+}$, and UCR-IBS ${ }^{-}$groups. ${ }^{*} P<0.05$. CD Crohn's disease, CDR Crohn's disease in remission, $C D R$-IBS ${ }^{+}$CDR with IBS-type symptoms, CDR-IBS- CDR without IBS-type symptoms, UC ulcerative colitis, UCR ulcerative colitis in remission, UCR-IBS ${ }^{+}$UCR with IBS-type symptoms, UCR-IBS- UCR without IBS-type symptoms

[27]. The greater richness and diversity of microbiota are seen as an indicator of good health, while decreased diversity and imbalance in microbiota may be closely related to a large range of diseases, especially intestinal diseases. Studies have confirmed that disorders of intestinal bacteria are involved in the occurrence and development of intestinal diseases, including IBS and IBD [1]. Partial IBD patients in remission may also suffer IBS-like symptoms, which may be related to the intestinal microbiota [28]. However, the results of our cross-sectional study indicate that the onset of IBS might not be related to obvious alterations in intestinal bacteria. Further analysis revealed that CD patients in remission with IBS-type symptoms might be related to an increase in Faecalibacterium and a decrease in Fusobacterium. There was no statistically significant difference in the abundance of intestinal bacteria between the UCR-IBS ${ }^{+}$and UCR-IBS ${ }^{-}$ groups at any taxonomic level. UC patients in remission with IBS-type symptoms cannot be explained by changes in the abundance and structure of the intestinal microbiota from our cross-sectional study.

Our study found that the proportion of Bacteroidetes in IBS patients at the phylum level tended to increase, while Firmicutes decreased compared with control subjects, but the difference was not statistically significant. At the genus level, the alterations in bacterial composition in
IBS patients also did not apparently differ from those in controls, while this result was not inconsistent with parts of previous research. Indeed, the role of the fecal microbiota in IBS is still controversial due to different sample sources, study populations, dietary habits and environmental factors. A recent prospective study comparing 110 IBS patients and 39 healthy controls demonstrated that the diversity of fecal microbiota and the number of Prevotella were reduced in IBS patients [29]. A systematic review involving microbiota in IBS revealed that the genus Bacteroides was increased in IBS patients compared with controls [30]. As shown in Fig. 3B, our results also showed an increasing trend of Bacteroides compared with the healthy controls, but the difference failed to achieve statistical significance, which might be explained by the different populations and sample sizes. Consistent with a previous study, our results also found no difference among major phyla or genera between IBS patients and controls [31]. Our results do not support a role for fecal microbiota in the pathogenesis of IBS and correlate with some other studies that reported significant differences between IBS patients and healthy controls in the composition of fecal microbiota $[11,32]$. The discrepancy may be explained by different populations, interindividual variation, and no further classification of IBS. Further 


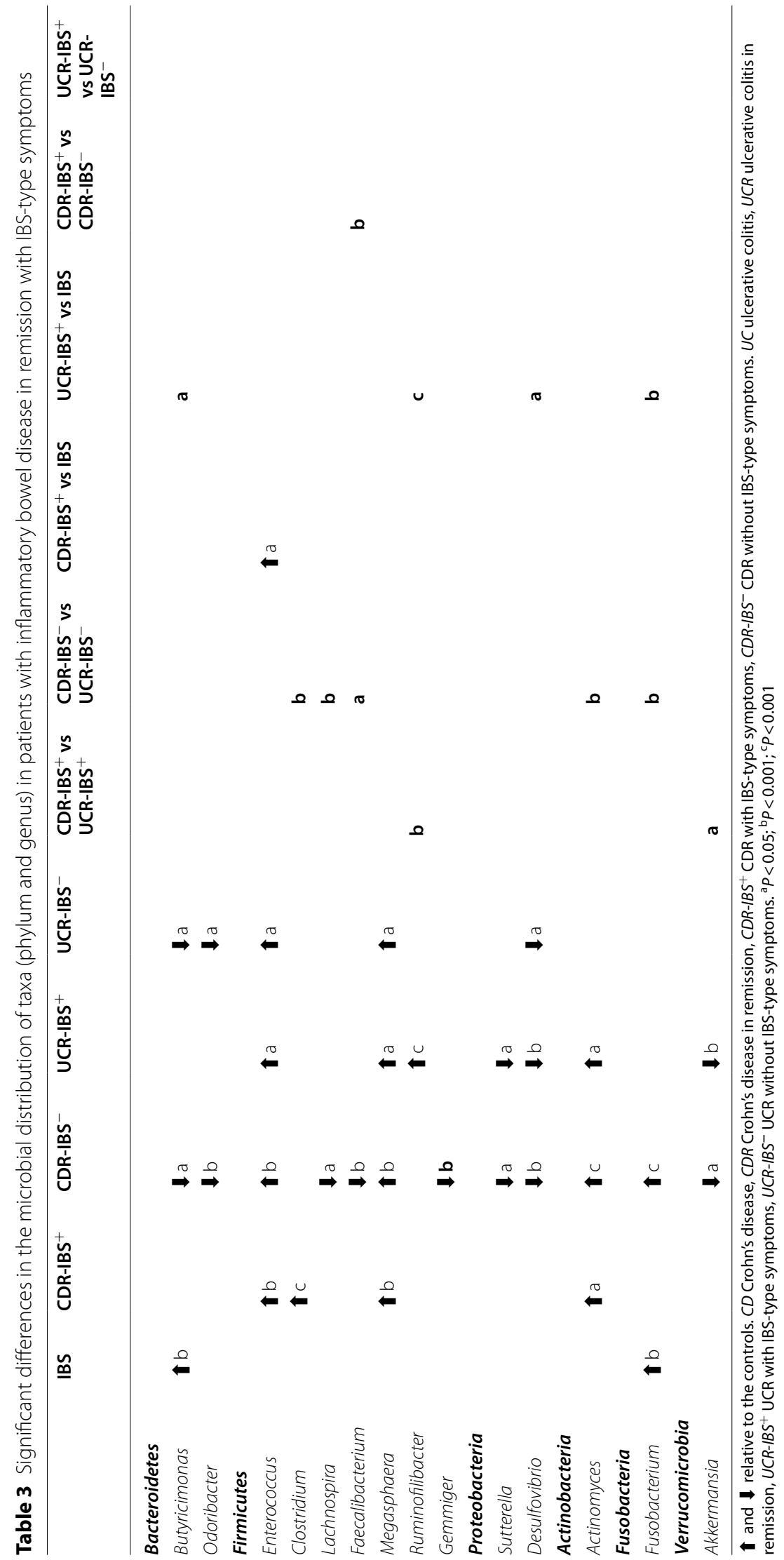


large-sample cohort studies are needed to confirm the characteristics of the fecal microbiota of IBS patients.

The interaction between the intestinal microbiota and enteric intestinal immune system is the general mechanism of the pathogenesis of IBD, especially in the active stage. Previous studies have confirmed that the disease activity of IBD patients is closely related to a decrease in anti-inflammatory bacterial species and an increase in pro-inflammatory bacterial species, as well as a decrease in overall alpha diversity [33]. However, some patients in IBD remission suffer from varying degrees of IBS-like symptoms [34, 35]. Is there any relationship between the intestinal flora of IBD patients with IBSlike symptoms and that of IBS patients? Therefore, we focused on exploring alterations in the microbiota community and diversity in IBDR patients with IBS-type symptoms. The results indicated that decreased richness (Chao1 and ACE index) was observed in the CDR-

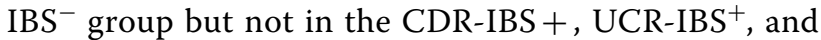
$\mathrm{UCR}^{-I_{B}}{ }^{-}$groups compared with the controls. Furthermore, there were trends toward a decreased number of Bacteroidetes and an increased number of Fusobacteria in the CDR-IBS ${ }^{+}$and CDR-IBS ${ }^{-}$groups based on the analysis of phylum taxonomic levels compared with the control and IBS groups, which might be due to the differences in the diseases. For genus taxonomic analysis, the bacterial community of the fecal sample from CDR-IBS $^{-}$exhibited an apparent difference from other groups due to the markedly higher number of Fusobacterium, an increasing trend of Escherichia and lower numbers of Lachnospira and Faecalibacterium compared to those in CDR-IBS ${ }^{+}$. However, there were no differences in richness and diversity across the CDR$\mathrm{IBS}^{+}$and $\mathrm{CDR}^{-\mathrm{IBS}^{-}}$groups or between the UCR-IBS ${ }^{+}$ and UCR-IBS ${ }^{-}$groups. A recent study conducted in IBS subjects showed that Fusobacterium might exacerbate visceral hypersensitivity [36], which is not consistent with our study. It may be that the study focused on diarrhea-predominant IBS (IBS-D), and we focused on the relationship between IBS symptoms and microbiota during IBD remission. In addition, Faecalibacterium belongs to butyrate-producing genera [37] and is elevated in fecal samples of patients with functional bowel disease. Consistent with previous studies, the relative abundance of Faecalibacterium was significantly higher than that in the CDR-IBS ${ }^{+}$group, indicating that the types of genera might play an important role in the formation of IBS-type symptoms. To date, our current results could not provide insight that there is a possible association between the presence of IBS-type symptoms in CD or UC patients in remission. Therefore, it is impossible to comment on any causal relationship between specific microbiome characteristics and the development of IBS-type symptoms, which is consistent with a previous study [21].

Of course, this study had certain limitations. First, this was a cross-sectional study and did not compare the dynamic changes in intestinal bacteria in the development of IBS-type symptoms in IBD patients. In addition, the associated microbiota in mucosa may more accurately reflect the relationship between microbiota and disease, but in our study, only fecal microbiota was detected and analyzed. Second, IBS is clinically divided into several types, including constipation (IBS-C), diarrhea (IBS-D), or a combination of both (IBS-mixed), according to the Rome IV Diagnostic Criteria [38]. The pathogenesis characteristics of the different types of IBS are somewhat different. However, due to the small sample size in this single-center study, no subgroup analysis was performed on the types of confirmed IBS patients or IBS-type symptoms. In addition, this study used the CDAI and Mayo scores to define the active and remission stages of $C D$ and $U C$, respectively, which are not the gold standards for intestinal inflammation. This is a possible reason for our inability to account for the significant alteration of microbiota in CDR-IBS ${ }^{-}$.

Our study and a previous study [21] failed to detect any difference in CDR-IBS composition and diversity in IBDR patients reporting IBS-type symptoms. However, clinical trials including probiotics and low fermentable oligosaccharide, disaccharide, monosaccharide, and polyol (FODMAP) diets have obtained promising results in IBS, indicating the role of the intestinal microbiota $[39,40]$. Additionally, the effects of probiotics and fecal bacteria transplantation (FMT) in the treatment of IBD have multiple benefits, and no attention is focused on the impact of these treatments on IBD-like symptoms $[41,42]$.

\section{Conclusions}

In conclusion, the obtained results from our study did not find any difference in the intestinal microbiota between IBD patients in remission with IBS-type symptoms and those without IBS-type symptoms. These results provide a certain basis for recommendations for future trials on the management of IBS-type symptoms. In the future, we still need to have a better understanding of the mechanism of IBD with IBS-type symptoms in the absence of persistent disease activity and strive to find effective treatments to relieve clinical symptoms and improve life treatment. 


\section{Supplementary Information}

The online version contains supplementary material available at https://doi. org/10.1186/s12876-021-02015-w.

Additional file 1: Figure S1. Rarefaction analysis of sampling by observed bacterial.

\section{Acknowledgements}

The authors appreciate the technical and statistical support of BGI Tech Solutions Co., Ltd. (Shenzhen, China) and would like to express their thanks.

\section{Authors' contributions}

HJZ, XFC and HYW designed the study and developed the concept. XFC and HYW performed the experiments and the statistical analyses. XFC wrote the manuscript. ZPY, XYQ collected the fecal samples. YL helped to modify the manuscript. HJZ revised and finalized the manuscript. HJZ supervised the report. All authors read and approved the final manuscript.

\section{Funding}

This work was supported by the National Natural Science Foundation of China (81770553) and the Postgraduate Research \& Practice Innovation Program of Jiangsu Province (JX10213640).

\section{Availability of data and materials}

The original data supporting this research conclusion are available from the corresponding author.

\section{Declarations}

\section{Ethics approval and consent to participate}

The study protocol and procedure were approved by the Ethics Committee of the First Affiliated Hospital of Nanjing Medical University, and informed consent was obtained from all enrolled participants before data and sample collection (Ethics number: 2018-SR-061). The study was carried out in accordance with the institutional guidelines.

\section{Consent for publication}

Not applicable.

\section{Competing interests}

The authors declare that they have no competing interests.

\section{Author details}

'Department of Gastroenterology, First Affiliated Hospital of Nanjing Medical University, 300\# Guangzhou Road, Nanjing 210029, Jiangsu Province, People's Republic of China. ${ }^{2}$ Department of Gastroenterology, Nanjing First Hospital, Nanjing Medical University, Nanjing, Jiangsu Province, People's Republic of China. ${ }^{3}$ Department of Gastroenterology, The Affiliated Sir Run Hospital, Nanjing Medical University, Nanjing 211100, Jiangsu Province, People's Republic of China.

Received: 21 January 2021 Accepted: 1 November 2021

Published online: 19 November 2021

\section{References}

1. Lo PA, Zorzi F, Del CF, Altomare A, Cocca S, Avola A, De Biasio F, Russo A, Cella E, Reddel S, et al. Fecal and mucosal microbiota profiling in irritable bowel syndrome and inflammatory bowel disease. Front Microbiol. 2019:10:1655.

2. Podolsky DK. Inflammatory bowel disease. N Engl J Med. 2002;347(6):417-29.

3. Yu LC. Microbiota dysbiosis and barrier dysfunction in inflammatory bowel disease and colorectal cancers: exploring a common ground hypothesis. J Biomed Sci. 2018;25(1):79.

4. Sokol H, Pigneur B, Watterlot L, Lakhdari O, Bermudez-Humaran LG, Gratadoux JJ, Blugeon S, Bridonneau C, Furet JP, Corthier G, et al.
Faecalibacterium prausnitzii is an anti-inflammatory commensal bacterium identified by gut microbiota analysis of Crohn disease patients. Proc Natl Acad Sci U S A. 2008;105(43):16731-6.

5. Liu S, Zhao W, Lan P, Mou X. The microbiome in inflammatory bowel diseases: from pathogenesis to therapy. Protein Cell. 2020;12:331-45.

6. Hoekman DR, Zeevenhooven J, D'Haens GR, Benninga MA. The prevalence of irritable bowel syndrome-type symptoms in inflammatory bowel disease patients in remission. Eur J Gastroenterol Hepatol. 2017;29(9):1086-90.

7. Keohane J, O'Mahony C, O'Mahony L, O'Mahony S, Quigley EM, Shanahan F. Irritable bowel syndrome-type symptoms in patients with inflammatory bowel disease: a real association or reflection of occult inflammation? Am J Gastroenterol. 2010;105(8):1789-94.

8. Sainsbury A, Sanders DS, Ford AC. Prevalence of irritable bowel syndrome-type symptoms in patients with celiac disease: a meta-analysis. Clin Gastroenterol Hepatol. 2013;11(4):359-65.

9. Halpin SJ, Ford AC. Prevalence of symptoms meeting criteria for irritable bowel syndrome in inflammatory bowel disease: systematic review and meta-analysis. Am J Gastroenterol. 2012;107(10):1474-82.

10. Gracie DJ, Williams CJ, Sood R, Mumtaz S, Bholah MH, Hamlin PJ, Ford AC. Negative effects on psychological health and quality of life of genuine irritable bowel syndrome-type symptoms in patients with inflammatory bowel disease. Clin Gastroenterol Hepatol. 2017;15(3):376-84.

11. Kassinen A, Krogius-Kurikka L, Makivuokko H, Rinttila T, Paulin L, Corander J, Malinen E, Apajalahti J, Palva A. The fecal microbiota of irritable bowel syndrome patients differs significantly from that of healthy subjects. Gastroenterology. 2007;133(1):24-33.

12. Chung CS, Chang PF, Liao CH, Lee TH, Chen Y, Lee YC, Wu MS, Wang HP, Ni $\mathrm{YH}$. Differences of microbiota in small bowel and faeces between irritable bowel syndrome patients and healthy subjects. Scand J Gastroenterol. 2016;51(4):410-9.

13. Labus JS, Hollister EB, Jacobs J, Kirbach K, Oezguen N, Gupta A, Acosta J, Luna RA, Aagaard K, Versalovic J, et al. Differences in gut microbial composition correlate with regional brain volumes in irritable bowel syndrome. Microbiome. 2017;5(1):49.

14. Menees S, Chey W. The gut microbiome and irritable bowel syndrome. F1000Res. 2018;7:1029.

15. Dupont HL. Review article: evidence for the role of gut microbiota in irritable bowel syndrome and its potential influence on therapeutic targets. Aliment Pharmacol Ther. 2014;39(10):1033-42.

16. Ghoshal UC, Srivastava D. Irritable bowel syndrome and small intestinal bacterial overgrowth: meaningful association or unnecessary hype. World J Gastroenterol. 2014;20(10):2482-91.

17. Magro F, Gionchetti P, Eliakim R, Ardizzone S, Armuzzi A, Barreiro-de AM, Burisch J, Gecse KB, Hart AL, Hindryckx P, et al. Third European Evidencebased Consensus on Diagnosis and Management of Ulcerative Colitis. Part 1: definitions, diagnosis, extra-intestinal manifestations, pregnancy, cancer surveillance, surgery, and ileo-anal pouch disorders. J Crohns Colitis. 2017;11(6):649-70.

18. Weimers P, Burisch J. The importance of detecting irritable bowel-like symptoms in inflammatory bowel disease patients. J Crohns Colitis. 2018;12(4):385-6.

19. Quigley EM, Fried M, Gwee KA, Khalif I, Hungin AP, Lindberg G, Abbas Z, Fernandez LB, Bhatia SJ, Schmulson M, et al. World gastroenterology organisation global guidelines irritable bowel syndrome: a global perspective update September 2015. J Clin Gastroenterol. 2016;50(9):704-13.

20. Gracie DJ, Ford AC. Irritable bowel syndrome-type symptoms are associated with psychological comorbidity, reduced quality of life, and health care use in patients with inflammatory bowel disease. Gastroenterology. 2017;153(1):324-5.

21. Shutkever O, Gracie DJ, Young C, Wood HM, Taylor M, John HP, Ford AC, Quirke P. No Significant association between the fecal microbiome and the presence of irritable bowel syndrome-type symptoms in patients with quiescent inflammatory bowel disease. Inflamm Bowel Dis. 2018;24(7):1597-605.

22. Claesson MJ, Wang Q, O'Sullivan O, Greene-Diniz R, Cole JR, Ross RP, O'Toole PW. Comparison of two next-generation sequencing technologies for resolving highly complex microbiota composition using tandem variable 16S rRNA gene regions. Nucleic Acids Res. 2010;38(22):e200.

23. Magoc T, Salzberg SL. FLASH: fast length adjustment of short reads to improve genome assemblies. Bioinformatics. 2011;27(21):2957-63. 
24. Bokulich NA, Subramanian S, Faith JJ, Gevers D, Gordon JI, Knight R, Mills DA, Caporaso JG. Quality-filtering vastly improves diversity estimates from Illumina amplicon sequencing. Nat Methods. 2013;10(1):57-9.

25. Nishida A, Inoue R, Inatomi O, Bamba S, Naito Y, Andoh A. Gut microbiota in the pathogenesis of inflammatory bowel disease. Clin J Gastroenterol. 2018;11(1):1-10.

26. Jandhyala SM, Talukdar R, Subramanyam C, Vuyyuru H, Sasikala M, Nageshwar RD. Role of the normal gut microbiota. World J Gastroenterol. 2015:21(29):8787-803.

27. O'Hara AM, Shanahan F. The gut flora as a forgotten organ. EMBO REP. 2006;7(7):688-93.

28. Chang BW, Rezaie A. Irritable bowel syndrome-like symptoms following fecal microbiota transplantation: a possible donor-dependent complication. Am J Gastroenterol. 2017;112(1):186-7.

29. Tap J, Derrien M, Tornblom H, Brazeilles R, Cools-Portier S, Dore J, Storsrud S, Le Neve B, Ohman L, Simren M. Identification of an intestinal microbiota signature associated with severity of irritable bowel syndrome. Gastroenterology. 2017;152(1):111-23.

30. Pittayanon R, Lau JT, Yuan Y, Leontiadis GI, Tse F, Surette M, Moayyedi P. Gut microbiota in patients with irritable bowel syndrome-a systematic review. Gastroenterology. 2019;157(1):97-108.

31. Dlugosz A, Winckler B, Lundin E, Zakikhany K, Sandstrom G, Ye W, Engstrand L, Lindberg G. No difference in small bowel microbiota between patients with irritable bowel syndrome and healthy controls. Sci Rep. 2015;5:8508.

32. Krogius-Kurikka L, Lyra A, Malinen E, Aarnikunnas J, Tuimala J, Paulin L, Makivuokko H, Kajander K, Palva A. Microbial community analysis reveals high level phylogenetic alterations in the overall gastrointestinal microbiota of diarrhoea-predominant irritable bowel syndrome sufferers. BMC Gastroenterol. 2009;9:95.

33. Gevers D, Kugathasan S, Denson LA, Vazquez-Baeza Y, Van Treuren W, Ren B, Schwager E, Knights D, Song SJ, Yassour M, et al. The treatmentnaive microbiome in new-onset Crohn's disease. Cell Host Microbe. 2014;15(3):382-92.

34. Ishihara S, Kawashima K, Fukuba N, Tada Y, Kotani S, Mishima Y, Oshima N, Kinoshita Y. Irritable bowel syndrome-like symptoms in ulcerative colitis patients in clinical remission: association with residual colonic inflammation. Digestion. 2019;99(1):46-51.

35. Ozer M, Bengi G, Colak R, Cengiz O, Akpinar H. Prevalence of irritable bowel syndrome-like symptoms using Rome IV criteria in patients with inactive inflammatory bowel disease and relation with quality of life. Medicine (Baltimore). 2020;99(19):e20067.

36. Gu X, Song $\sqcup$, Li LX, Liu T, Zhang MM, Li Z, Wang P, Li M, Zuo XL. Fusobacterium nucleatum causes microbial dysbiosis and exacerbates visceral hypersensitivity in a colonization-independent manner. Front Microbiol. 2020;11:1281

37. Louis P, Flint HJ. Diversity, metabolism and microbial ecology of butyrateproducing bacteria from the human large intestine. FEMS Microbiol LETT. 2009:294(1):1-8.

38. Mearin F, Lacy BE, Chang L, Chey WD, Lembo AJ, Simren M, Spiller R. Bowel disorders. Gastroenterology. 2016;150:1393-407.

39. Ford AC, Quigley EM, Lacy BE, Lembo AJ, Saito YA, Schiller LR, Soffer EE, Spiegel BM, Moayyedi P. Efficacy of prebiotics, probiotics, and synbiotics in irritable bowel syndrome and chronic idiopathic constipation: systematic review and meta-analysis. Am J Gastroenterol. 2014;109(10):1547-61 .

40. Staudacher HM, Lomer M, Farquharson FM, Louis P, Fava F, Franciosi E, Scholz M, Tuohy KM, Lindsay JO, Irving PM, et al. A diet low in FODMAPs reduces symptoms in patients with irritable bowel syndrome and a probiotic restores bifidobacterium species: a randomized controlled trial. Gastroenterology. 2017;153(4):936-47.

41. Nitzan O, Elias M, Peretz A, Saliba W. Role of antibiotics for treatment of inflammatory bowel disease. World J Gastroenterol. 2016;22(3):1078-87.

42. Vermeire S, Joossens M, Verbeke K, Wang J, Machiels K, Sabino J, Ferrante M, Van Assche G, Rutgeerts P, Raes J. Donor species richness determines faecal microbiota transplantation success in inflammatory bowel disease. J Crohns Colitis. 2016;10(4):387-94.

\section{Publisher's Note}

Springer Nature remains neutral with regard to jurisdictional claims in published maps and institutional affiliations.
Ready to submit your research? Choose BMC and benefit from:

- fast, convenient online submission

- thorough peer review by experienced researchers in your field

- rapid publication on acceptance

- support for research data, including large and complex data types

- gold Open Access which fosters wider collaboration and increased citations

- maximum visibility for your research: over $100 \mathrm{M}$ website views per year

At BMC, research is always in progress.

Learn more biomedcentral.com/submissions 